TRANSACTIONS OF THE

AMERICAN MATHEMATICAL SOCIETY

Volume 358, Number 8, Pages 3447-3457

S 0002-9947(06)04144-4

Article electronically published on March 1, 2006

\title{
EXPLICIT BOUNDS FOR THE FINITE JET DETERMINATION PROBLEM
}

\author{
BERNHARD LAMEL
}

\begin{abstract}
We introduce biholomorphic invariants for (germs of) rigid holomorphically nondegenerate real hypersurfaces in complex space and show how they can be used to compute explicit bounds on the order of jets for which biholomorphisms of the hypersurface are determined uniquely by their jets. The main result which allows us to derive these bounds is a theorem which shows that solutions of certain singular analytic equations are uniquely determined by their 1 -jet.
\end{abstract}

\section{INTRODUCTION}

The work on determination of holomorphic maps between real submanifolds of complex spaces by jets of finite order probably starts with Poincaré's famous paper 12. Although we will not give a historical introduction here (the survey article by Zaitsev [16] is recommended to the interested reader), we do want to mention some results which help put our theorems in context.

The following notation will be used throughout this paper: Given two germs $\left(M, p_{0}\right)$ and $\left(M^{\prime}, p_{0}^{\prime}\right)$ of real analytic hypersurfaces in $\mathbb{C}^{N}$ and a germ of a biholomorphism $H$ of $\mathbb{C}^{N}$ defined in a neighbourhood $U$ of $p_{0}$ which satisfies $H\left(p_{0}\right)=p_{0}^{\prime}$ and for some neighbourhood $V \subset U$ of $p, H(M \cap V) \subset M^{\prime}$, we write $H:\left(M, p_{0}\right) \rightarrow$ $\left(M^{\prime}, p_{0}^{\prime}\right)$.

Given germs of real analytic hypersurfaces $\left(M, p_{0}\right)$ at $p_{0}$ and $\left(M^{\prime}, p_{0}^{\prime}\right)$ at $p_{0}^{\prime}$, respectively, we say that the biholomorphisms from $\left(M, p_{0}\right)$ to $\left(M^{\prime}, p_{0}^{\prime}\right)$ are determined by their jets of order $k$ if any two (germs of) biholomorphisms $H, \tilde{H}:\left(M, p_{0}\right) \rightarrow$ $\left(M^{\prime}, p_{0}^{\prime}\right)$ with

$$
\frac{\partial^{|\alpha|} H}{\partial Z^{\alpha}}\left(p_{0}\right)=\frac{\partial^{|\alpha|} \tilde{H}}{\partial Z^{\alpha}}\left(p_{0}\right), \quad|\alpha| \leq k,
$$

actually agree, that is, $H(Z)=\tilde{H}(Z)$ for all $Z$ in a neighbourhood of $p_{0}$.

If we write $\operatorname{Aut}\left(M, p_{0}\right)$ for the group of automorphisms of $M$, that is, biholomorphisms $H:\left(M, p_{0}\right) \rightarrow\left(M, p_{0}\right)$, this question is equivalent to studying the injectivity of the map which associates to a map $H \in \operatorname{Aut}\left(M, p_{0}\right)$ its $k$-jet. Actually, if the automorphisms of $\left(M, p_{0}\right)$ are determined by their $k$-jets, so are the biholomorphisms from $\left(M, p_{0}\right)$ to $\left(M^{\prime}, p_{0}^{\prime}\right)$ for all $\left(M^{\prime}, p_{0}^{\prime}\right)$ (this is easy to see by composing with a fixed biholomorphism; see [10]).

The first class of hypersurfaces for which finite jet determination results were established is the class of Levi-nondegenerate hypersurfaces. These results are due

Received by the editors May 17, 2004.

2000 Mathematics Subject Classification. Primary 32H02.

The author was supported by the FWF, Projekt P15279 and P17111.

(C)2006 American Mathematical Society 3447

Reverts to public domain 28 years from publication 
to Cartan [7], $[8$ in the 2-dimensional case and, for higher dimensions, to Chern and Moser [9] and Tanaka [14. They prove that for Levi-nondegenerate hypersurfaces biholomorphisms are already determined by their 2-jets. This bound is sharp since maps between quadrics depend on their second order jet.

The next class of hypersurfaces for which finite jet determination was proved is the class of finitely nondegenerate hypersurfaces (introduced by Baouendi, Huang and Rothschild [4]). Here, the result (due to Baouendi, Ebenfelt and Rothschild [1]) is that for an $\ell$-nondegenerate hypersurface the maps are determined by the jets of order $2 \ell$. However, we know that this bound, in contrast to the Levi-nondegenerate case, is not sharp.

Finite jet determination was also proved for the class of essentially finite hypersurfaces by Baouendi, Ebenfelt and Rothschild [2]. However, for this class we do not know any good bounds on $k$ yet.

One of the results of Baouendi, Mir and Rothschild [5] is that if $\left(M, p_{0}\right)$ is holomorphically nondegenerate (that is, if there does not exist a germ of a holomorphic $(1,0)$-vector field at $p_{0}$ tangent to $M$ ) and of finite type, then finite jet determination also holds. This result completely solves the problem in the finite type case, as it is known that holomorphic nondegeneracy is necessary for finite determination, apart from the question of how big $k$ is.

Motivated by these results, we study the question of how $k$ is determined by geometric invariants of $M$. We have already cited some results in this direction above. Another result, due to Ebenfelt, Zaitsev and myself [10, is that for a finite type hypersurface in $\mathbb{C}^{2}, k=2$ suffices. It is not true, though, that for $N>2$, automorphisms of hypersurfaces (even of finitely nondegenerate ones) in $\mathbb{C}^{N}$ are determined by a jet whose order does only depend on $N$, as the following example shows.

Example 1. For $\ell \geq 2$, let $M \subset \mathbb{C}^{3}$ be the real hypersurface defined by

$$
\operatorname{Im} w=\left|z_{1}\right|^{2}+\operatorname{Re} z_{1}^{\ell} \bar{z}_{2},
$$

where $\left(z_{1}, z_{2}, w\right)$ are the coordinates in $\mathbb{C}^{3} . M$ is of finite type and finitely nondegenerate at 0 . For any $a \in \mathbb{R}$, the polynomial automorphism

$$
H_{a}\left(z_{1}, z_{2}, w\right)=\left(z_{1}, z_{2}+i a z_{1}^{\ell}, w\right)
$$

sends $(M, 0)$ into itself, and its $(\ell-1)$-jet at 0 coincides with that of the identity.

In this paper, we will only discuss the case of a rigid hypersurface, that is, a hypersurface which can be defined by an equation of the form

$$
w-\bar{w}=q(z, \bar{z}), \quad(z, w) \in \mathbb{C}^{n} \times \mathbb{C}=\mathbb{C}^{N} .
$$

Even though many of the considerations in the following sections apply to more general classes of hypersurfaces, we limit the discussion to this case, as it is more tractable and the results for the rigid case are distinct. Our main result improves the known results for this special case. The invariants $m_{0}, m_{1}$ and $\ell$ used in this theorem will be introduced below and are relatively easy to compute $\left(m_{0}\right.$ and $m_{1}$ are defined in Definition 4, and $\ell$, defined in (6) below, is actually the Levi-number of $M)$.

Theorem 1. Let $\left(M, p_{0}\right),\left(M^{\prime}, p_{0}^{\prime}\right)$ be germs of rigid, holomorphically nondegenerate real-analytic hypersurfaces in $\mathbb{C}^{N}$. If $H$ and $\tilde{H}$ are germs of biholomorphisms 
taking $\left(M, p_{0}\right)$ to $\left(M^{\prime}, p_{0}^{\prime}\right)$ and

$$
\frac{\partial^{|\alpha|} H}{\partial Z^{\alpha}}\left(p_{0}\right)=\frac{\partial^{|\alpha|} \tilde{H}}{\partial Z^{\alpha}}\left(p_{0}\right), \quad|\alpha| \leq m_{1}-m_{0}+\ell+1
$$

then $H=\tilde{H}$.

The bound in this theorem is actually sharp for some special classes of hypersurfaces which will be mentioned in the text (see Example 4 below). However, in general we do not know whether the bound provided by Theorem 1 is optimal.

Our argument is based on the solution of a quite different form of 1-jet determination problem: Assume that $A(z)=\left(A_{1}(z), \ldots, A_{n}(z)\right)$ is a germ of a generically finite mapping $\left(\mathbb{C}^{n}, 0\right) \rightarrow\left(\mathbb{C}^{n}, 0\right)$. Recall that generically finite means that $\operatorname{det} A^{\prime}(z) \neq 0$ as elements of $\mathbb{C}\{z\}$. If we solve an equation of the form $A(X)=b(z)$ for $X$ in terms of $z$, where we only allow automorphisms $\left(\mathbb{C}^{n}, 0\right) \rightarrow\left(\mathbb{C}^{n}, 0\right)$ as solutions, how unique are the solutions? The answer is provided by the following theorem.

Theorem 2. Let $A(z)=\left(A_{1}(z), \ldots, A_{n}(z)\right)$ be a germ of a generically finite mapping $\left(\mathbb{C}^{n}, 0\right) \rightarrow\left(\mathbb{C}^{n}, 0\right)$. If $u(z):\left(\mathbb{C}^{n}, 0\right) \rightarrow\left(\mathbb{C}^{n}, 0\right)$ and $v(z):\left(\mathbb{C}^{n}, 0\right) \rightarrow\left(\mathbb{C}^{n}, 0\right)$ are germs of biholomorphisms with $A(u(z))=A(v(z))$ and $u^{\prime}(0)=v^{\prime}(0)$, then $u=v$.

This theorem is the technical heart of this paper. The proof is given in Section 3. Actually, Theorem 2 is well known and easy to prove in the case of a finite mapping $A$, which can also take values in a higher-dimensional space $\mathbb{C}^{k}, k>n$. This version actually also follows from the theorem above.

\section{Holomorphic Nondegeneracy and SEgre varieties}

Let us first recall the notion of normal coordinates for a real analytic hypersurface. Coordinates $Z=(z, w)=\left(z_{1}, \ldots, z_{n}, w\right) \in \mathbb{C}^{n} \times \mathbb{C}$ in $\mathbb{C}^{N}$ are called normal coordinates for $M$ at $p_{0}$ if in these coordinates $p_{0}=0$ and if there exists a holomorphic function $Q(z, \chi, \tau)$ satisfying $Q(z, 0, \tau)=Q(0, \chi, \tau)=\tau$ defined in a neighbourhood of $0 \in \mathbb{C}^{2 n+1}$ such that for some neighbourhood $U$ of $0 \in \mathbb{C}^{N}$,

$$
M \cap U=\{Z \in U: w=Q(z, \bar{z}, \bar{w})\} .
$$

$M$ is then also given by $\bar{w}=\bar{Q}(\bar{z}, z, w)$, and $Q$ satisfies the reality condition $Q(z, \chi, \bar{Q}(\chi, z, w))=w$.

If $M$ is a rigid hypersurface given by $w-\bar{w}=q(z, \bar{z})$, then $(z, w)$ are normal coordinates for $M$ if $q$ satisfies $q(z, 0)=q(0, \chi)=0$.

Following Stanton [13, a germ of a real analytic hypersurface $\left(M, p_{0}\right)$ is holomorphically nondegenerate if there does not exist a germ of a nontrivial holomorphic $(1,0)$-vector field tangent to $M$, that is, if there does not exist a vector field

$$
X=\sum_{j} a_{j}(Z) \frac{\partial}{\partial Z_{j}}, \quad a_{j} \in \mathcal{O}_{p_{0}},
$$

which is tangent to $M$ near $p_{0}$.

It is well known (a proof of this fact can be found in the paper of Baouendi and Rothschild [6]) that $\left(M, p_{0}\right)$ is holomorphically nondegenerate if and only if, writing $\bar{Q}(\chi, Z)=\sum_{\alpha} b_{\alpha}(Z) \chi^{\alpha}$, there exist $\alpha_{1}, \ldots, \alpha_{n}$ such that the mapping $\left(b_{\alpha_{1}}, \ldots, b_{\alpha_{n}}\right)$ is generically finite. 
In particular, a rigid hypersurface given by $w-\bar{w}=q(z, \bar{z})=\sum_{\alpha} q_{\alpha}(\bar{z}) z^{\alpha}$ is holomorphically nondegenerate if and only if there exist $\alpha_{1}, \ldots, \alpha_{n}$ such that the mapping $\left(q_{\alpha_{1}}, \ldots, q_{\alpha_{n}}\right)$ is generically finite.

We will need a numerical invariant associated to holomorphic nondegeneracy in this paper. Since this invariant can be defined for a general real analytic hypersurface, we will not restrict ourselves to the rigid case here.

To prepare for the definition, we recall that if $M$ is given in normal coordinates by $w=Q(z, \bar{z}, \bar{w}), M^{\prime}$ similarly by $w^{\prime}=Q^{\prime}\left(z^{\prime}, \bar{z}^{\prime}, \bar{w}^{\prime}\right)$ and $H=(f, g)$ is a biholomorphic map, then

$$
\begin{aligned}
Q^{\prime}(f(z, Q(z, \chi, \tau)), \bar{f}(0, Q(z, \chi, \tau)), \bar{g}(0, Q(z, \chi, \tau))) & \\
& =Q^{\prime}(f(z, Q(z, \chi, \tau)), \bar{f}(\chi, \tau), \bar{g}(\chi, \tau)) .
\end{aligned}
$$

We write $Q(z, \chi, \tau)=\tau+\sum q_{\alpha, \mu}(\chi) z^{\alpha} \tau^{\mu}$, and we also write $q_{\alpha, 0}=q_{\alpha}$, with the analog notation for $Q^{\prime}$. Setting $\tau=0$ in (1), we then obtain

$$
\begin{aligned}
\bar{g}(0, Q(z, \chi, 0))= & \sum q_{\alpha}^{\prime}(\bar{f}(\chi, 0)) f(z, Q(z, \chi, 0))^{\alpha} \\
& -\sum q_{\alpha, \mu}^{\prime}(\bar{f}(0, Q(z, \chi, 0))) f(z, Q(z, \chi, 0))^{\alpha} \bar{g}(0, Q(z, \chi, 0))^{\mu} .
\end{aligned}
$$

In the next lemma, we introduce a sequence of rings, which are invariantly attached to $(M, 0)$. We write $q(\chi)=\left(q_{e_{1}}(\chi), \ldots, q_{e_{n}}(\chi)\right)$.

Lemma 3. Let $(M, 0),\left(M^{\prime}, 0\right)$ be germs of real-analytic hypersurfaces in $\mathbb{C}^{N},(z, w)$ normal coordinates for $M,\left(z^{\prime}, w^{\prime}\right)$ normal coordinates for $M^{\prime}$, so that $M$ is given by $w=Q(z, \bar{z}, \bar{w})$ and similarly $M^{\prime}$, and $H=(f, g):(M, 0) \rightarrow\left(M^{\prime}, 0\right)$ a biholomorphism. Define increasing chains of subrings $R_{k}, R_{k}^{\prime}$ by

$$
\begin{aligned}
& R_{0}=\mathbb{C}\{q(\chi)\} \subset \mathbb{C}\{\chi\}, \\
& R_{k}=R_{0}\left[q_{\alpha}(\chi):(|\alpha|) \leq k\right] \subset \mathbb{C}\{\chi\},
\end{aligned}
$$

with the analog notation for the objects attached to $M^{\prime}$. (Note by the way that if $m_{0} \neq 1, R_{0}=\mathbb{C}$.) Then

$$
R_{k}^{\prime} \circ \bar{f}(\chi, 0)=R_{k} .
$$

Proof. The statement for $k=0$ is left to the reader. Note that it suffices to prove $R_{k} \subset R_{k}^{\prime} \circ \bar{f}(\chi, 0)$, since $f(z, 0)$ is a germ of a biholomorphism in $\mathbb{C}^{n}$. If $k>0$, let us assume that $R_{k-1}=R_{k-1}^{\prime} \circ \bar{f}(\chi, 0)$. We examine the coefficient of $z^{\alpha}$, where $|\alpha|=k$, in (2). On the left-hand side, we obtain $\bar{g}_{w}(0) q_{\alpha}(\chi)$ and a polynomial in $q_{\beta}(\chi)$, where $\beta<k$. On the right-hand side, we get

$$
\sum_{\beta=k} q_{\beta}^{\prime}(\bar{f}(\chi, 0)) K_{\alpha}^{\beta}
$$

a polynomial in $q_{\beta}^{\prime}(\bar{f}(\chi, 0))$, where $\beta<k$, and a polynomial in $q_{\beta}$, where $\beta<$ $k$ (observe that $q_{\alpha, \mu}^{\prime}(0)=0$ ). We have used the notation $K_{\alpha}^{\beta}$ to stand for the coefficient of $z^{\alpha}$ in

$$
\prod_{l=1}^{n}\left(\sum_{j=1}^{n} f_{l, z_{j}}(0) z_{j}+f_{l, w}(0) q_{e_{j}}(\chi) z_{j}\right)^{\beta_{l}} .
$$


Hence, since $\left(K_{\alpha}^{\beta}\right)_{|\alpha|=|\beta|=k}$ is invertible in a neighbourhood of 0 , and the entries of the inverse are given by power series in $q(\chi), R_{k} \subset R_{k}^{\prime} \circ \bar{f}(\chi, 0)$, and the induction is finished.

We will use this lemma to introduce two invariants. Before we do that, we need one more notation. For a subring $R \subset \mathbb{C}\{\chi\}$, we denote by rk $R$ the maximal number of elements of $R$ with generically linearly independent differentials. Here, generically linearly independent is understood as independent over the field of fractions of $\mathbb{C}\{\chi\}$. We note that rk $R$ is the same as the generic rank of the differentials of any subset $S \subset \mathbb{C}\{\chi\}$ which generates $R$ as a subring. This is the reason why we have to deal with subrings here, as the following example shows.

Example 2. Consider the ideal $I=(z) \subset \mathbb{C}\{z, w\}$. Then the functions $z \in I$ and $z w \in I$ have generically linearly independent differentials, but $I$ is generated by only one function.

Definition 4. Let $(M, 0)$ be a germ of a real-analytic hypersurface in $\mathbb{C}^{N}$, and define $R_{k}$ by (3). We define

$$
m_{0}=\min \left\{k: R_{k} \neq \mathbb{C}\right\}, \quad m_{1}=\min \left\{k: \operatorname{rk} R_{k}=n\right\} .
$$

We note that $1 \leq m_{0} \leq m_{1}, m_{0}<\infty$ if and only if $M$ is of finite type at 0 , and if $M$ is rigid, $m_{1}<\infty$ if and only if $M$ is holomorphically nondegenerate at 0 . It is also easy to see that if $M$ is $k$-nondegenerate at 0 , then $m_{1} \leq k$. In Example 1 . we have $m_{0}=1$ and $m_{1}=l$.

For our next invariant, we will first give a geometric description (for the expert reader, we note now that this invariant will be seen to be the Levi-number of $M$, which is formulated in Lemma [6 below). For a point $P \in \mathbb{C}^{N}$, denote by $E_{P}^{k}$ the space of $k$-jets of complex hypersurfaces passing through $P$, and denote by $e_{P}^{k}$ the mapping which to a germ of a complex hypersurface at $P$ associates its $k$-jet at $P$ (for more information on the jet spaces used here, we refer the reader to, e.g., the discussion in the paper of Zaitsev [15]). We write $S_{\zeta}$ for the Segre variety associated to $\zeta$, that is, the complex hypersurface given by $w=Q(z, \bar{\zeta})$ (the basic facts on Segre varieties used here can be found in the book [3]). For each $k \in \mathbb{N}$ and $Z \in S_{0}$ sufficiently close to $0, \Phi_{Z}^{k}: S_{Z} \rightarrow E_{Z}^{k}, \Phi_{Z}^{k}(\zeta)=e_{Z}^{k} S_{\zeta}$ defines a germ of an antiholomorphic map at 0 . We define

$$
s=\min \left\{k: \Phi_{Z}^{k} \text { is generically finite for generic } Z \in S_{0}\right\} .
$$

Our next lemma helps us understand this invariant in normal coordinates.

Lemma 5. Let $M$ be a rigid hypersurface given in normal coordinates near 0 by $w-\bar{w}=q(z, \chi)$. Write $R_{k}^{z}$ for the subring of $\mathbb{C}\{\chi\}$ generated by the set $\left\{\bar{q}_{z^{\alpha}}(\chi, z, 0): 1 \leq|\alpha| \leq k\right\}$. Then

$$
s=\min \left\{k: \operatorname{rk} R_{k}^{z}=n \text { for generic } z \in \mathbb{C}^{n}\right\} .
$$

In particular, if $M$ is holomorphically nondegenerate, then $s \leq m_{1}<\infty$.

Proof. We only need to unwind the definitions; it is understood that all points have to be restricted to lie close by 0 . First, if $M$ is given in normal coordinates, then the first Segre variety through 0 is just the complex hyperplane $w=0$. Thus, we have to compute the Segre varieties through points of the form $\left(z_{0}, 0\right)$. We have $S_{\left(z_{0}, 0\right)}=\left\{(\bar{\chi}, \bar{\tau}): \bar{\tau}=q\left(\bar{\chi}, \bar{z}_{0}\right)\right\}$. Thus, for $\zeta \in S_{\left(z_{0}, 0\right)}, S_{\zeta}=\{(z, w): w=$ $\left.q(z, \chi)+\bar{q}\left(\chi, z_{0}\right)\right\}$. This means that we are actually given a parametrization of $S_{\zeta}$ 
over the plane $w=0$, and we can choose coordinates in $E_{\left(z_{0}, 0\right)}^{k}$ by declaring that $e_{z_{0}}^{k}\{w=\varphi(z)\}=\left(\varphi_{z^{\alpha}}\left(z_{0}\right)\right)_{|\alpha| \leq k}$. We choose $\bar{\chi}$ as the coordinate on $S_{\left(z_{0}, 0\right)}$. The mapping $\Phi_{\left(z_{0}, 0\right)}^{k}$ is thus given by

$$
\Phi_{\left(z_{0}, 0\right)}^{k}(\bar{\chi})=\left(q_{z^{\alpha}}\left(z_{0}, \chi\right)\right)_{1 \leq|\alpha| \leq k} .
$$

Example 3. Let $M$ be a hypersurface given by $\operatorname{Im} w=\sum_{j=1}^{k}\left|f_{j}(z)\right|^{2}$, where $f_{j} \in$ $\mathbb{C}\{z\}$ and the mapping $\left(f_{1}, \ldots, f_{k}\right)$ is generically finite (e.g., if the ideal generated by the $f_{j}$ has finite codimension). Then $s=1$ : Indeed, computing the mapping $\Phi_{z}^{1}$, we see that it is given by

$$
\Phi_{z}^{1}(\bar{\chi})=2 i \sum \bar{f}(\chi) f_{1, z}(z)
$$

so its Jacobian is given by

$$
2 i\left|\begin{array}{ccc}
f_{z_{1}}(z) \cdot f_{\chi_{1}}(\chi) & \ldots & f_{z_{1}}(z) \cdot f_{\chi_{n}}(\chi) \\
\vdots & & \vdots \\
f_{z_{n}}(z) \cdot f_{\chi_{1}}(\chi) & \ldots & f_{z_{n}}(z) \cdot f_{\chi_{n}}(\chi)
\end{array}\right|
$$

which is generically nonzero since by assumption $f_{z_{1}}, \ldots, f_{z_{n}}$ are generically independent.

Example 4. We can now also describe a class of hypersurfaces for which determination by 2 -jets is guaranteed by Theorem 1 and for which this is optimal. Indeed, consider the class of rigid hypersurfaces of the form

$$
w-\bar{w}=\sum_{|\alpha|=d} q_{\alpha}(\chi) z^{\alpha},
$$

where $q_{\alpha}$ is homogeneous of degree $d$ (that is, $q$ is bihomogeneous of degree $d$ ). A hypersurface of this form is holomorphically nondegenerate if and only if $\chi \mapsto$ $\left(q_{\alpha}(\chi)\right)_{|\alpha|=d}$ is generically finite. In this case, Theorem 1 implies that the biholomorphisms of such a hypersurface are determined by their 2-jets. The bound is realized for this particular class of hypersurfaces since they possess the one-parameter family of automorphisms given by

$$
z \mapsto \frac{z}{(1-t w)^{\frac{1}{d}}}, \quad w \mapsto \frac{w}{1-t w},
$$

which are determined by their 2-jets. A particular example is given by hypersurfaces of the form

$$
w-\bar{w}=2 i \sum_{\alpha}\left|z^{\alpha}\right|^{2}
$$

where the $\alpha$ range over a set of multi-indices containing multi-indices $\alpha^{j}=$ $\left(\alpha_{1}^{j}, \ldots, \alpha_{n}^{j}\right), j=1, \ldots, n$, with $\operatorname{det}\left(\alpha_{j}^{k}\right) \neq 0$.

Example 5. The light cone $M \subset \mathbb{C}^{3}$ given (in nonnormal coordinates) by $\left(\operatorname{Re} z_{1}\right)^{2}$ $+\left(\operatorname{Re} z_{2}\right)^{2}=\left(\operatorname{Re} z_{3}\right)^{2}$ has $s=2$, as the next lemma shows.

Let us recall that a holomorphically nondegenerate hypersurface is actually $\ell$ nondegenerate for some $\ell \leq n$ at all points outside a proper real-analytic subvariety of $M$. This number is called the Levi-number of $M$ and was introduced in the paper of Baouendi, Huang and Rothschild [4]. 
Lemma 6. Let $\left(M, p_{0}\right)$ be a germ of a rigid holomorphically nondegenerate hypersurface in $\mathbb{C}^{N}$. Then $s=\ell$, the Levi number of $M$.

The proof of this lemma is contained in the book of Baouendi, Ebenfelt and Rothschild [3] in Chapter XI, so we do not reproduce it here.

\section{Determination Along $S_{0}$}

We will now determine $H$ along $S_{0}$, which in normal coordinates is just the plane $w=0$. We now assume that $M$ is a rigid, holomorphically nondegenerate hypersurface. We will also only deal with automorphisms $H$ of this hypersurface; as noted in the Introduction, the more general results on determination of biholomorphisms follow from these.

Lemma 7. For $\alpha \in \mathbb{N}^{n},|\alpha| \geq m_{0}$, there exist rational functions $p_{\alpha}$ which only depend on $M$ such that

$$
q_{\beta}(\bar{f}(\chi, 0))=p_{\beta}\left(q, j_{0}^{k} H, j_{0}^{k} \bar{H}\right),
$$

where $k \leq|\alpha|-m_{0}+1$ and $q$ stands for $q_{\alpha}$ and its derivatives, evaluated at either $\chi$ or 0, for $|\alpha| \leq|\beta|$.

Proof. We extract the coefficient of $\chi^{\alpha}$ in (2), which for a rigid hypersurface we rewrite as

$$
\begin{aligned}
& \bar{g}(0, q(z, \chi, 0)) \\
= & \sum q_{\alpha}(\bar{f}(\chi, 0)) f(z, Q(z, \chi, 0))^{\alpha}-\sum q_{\alpha}(\bar{f}(0, Q(z, \chi, 0))) f(z, Q(z, \chi, 0))^{\alpha},
\end{aligned}
$$

using induction on $|\alpha|$. For $|\alpha|=m_{0}$, following the proof of Lemma 3 we see that

$$
g_{w}(0) q_{\alpha}(\chi)=\sum_{|\beta|=m_{0}} q_{\beta}(\bar{f}(\chi, 0)) K_{\alpha}^{\beta}(\chi)
$$

Now note that $K_{\alpha}^{\beta}(0)$ is an invertible matrix whose entries are (linear) polynomials in $j_{0}^{1} f$ and $q_{e_{j}}(\chi)$. Multiplying equation (12) by the inverse of $K_{\alpha}^{\beta}$, we obtain (10) for $|\alpha|=m_{0}$. For $|\gamma|=m>m_{0}$, we assume that we have (10) for $|\beta|<|\gamma|$. We now extract the coefficient of $z^{\gamma}$ in (2). On the left-hand side, developing in a Taylor series, we get

$$
\sum_{1 \leq \ell \leq\left[\frac{|\gamma|}{m_{0}}\right]} \frac{\bar{g}_{w^{\ell}}(0)}{\ell !}\left(\sum_{\gamma_{1}+\cdots+\gamma_{l}=\gamma} q_{\gamma_{1}}(\chi) \ldots q_{\gamma_{l}}(\chi)\right) .
$$

We note that since $m_{0} \geq 1, \ell \leq|\gamma|$, and so only derivatives of $g$ of the order claimed in the lemma appear; furthermore, only $q_{\beta}(\chi)$ for $|\beta| \leq|\gamma|$ appear in the sum.

Now let us turn to the right-hand side. We start with the first sum. Developing $f$ into a Taylor series, it is easy to see that only derivatives of order at most $|\gamma|$ can appear. Also, only $q_{\beta}(\bar{f}(\chi, 0))$ for $|\beta| \leq|\gamma|$ can appear. For those with $|\beta|<|\gamma|$, we use the induction hypothesis and replace them by the rational functions (10). The part with $|\beta|=|\gamma|$ is then

$$
\sum_{|\beta|=|\gamma|} q_{\beta}(\bar{f}(\chi, 0)) K_{\gamma}^{\beta}(\chi) .
$$


From the second sum on the right-hand side, it is easy to see that (after applying the induction hypothesis) only terms allowed in the $p_{\beta}$ enter. Hence, we have equations of the form

$$
\sum_{|\beta|=|\gamma|} q_{\beta}(\bar{f}(\chi, 0)) K_{\gamma}^{\beta}(\chi)=r_{\gamma}\left(q, j_{0}^{k} H, \bar{j}_{0}^{k} H\right), \quad|\gamma|=m .
$$

Applying the inverse matrix of $K_{\gamma}^{\beta}$, we obtain (10) for $|\beta|=m$, and the induction is finished.

For the next step, we will need to apply Theorem 2] so we include its proof here.

Proof of Theorem 2. By considering $u \circ v^{-1}$ we see that it is enough to prove the theorem for $v(z)=z$. We start by writing

$$
A(y)-A(x)=\int_{0}^{1} A^{\prime}(t y+(1-t) x) d t(y-x)=R(x, y)(y-x) .
$$

Setting $y=u(z), x=z$, we see that

$$
R(z, u(z))(u(z)-z)=0 .
$$

It is thus enough to show that under our assumptions, $\operatorname{det} R(z, u(z)) \neq 0$ (in $\mathbb{C}\{z\}$ ).

Expanding $A_{j}$ into homogeneous terms,

$$
A_{j}(z)=\sum_{k \geq k_{j}} A_{j, k}(z), \quad A_{j, k}(t z)=t^{k} A_{j, k}(z)
$$

we see that since $u(z)=z+O(2)$, $\operatorname{det} R(z, u(z)) \neq 0$ provided that

$$
\left|A_{1, k_{1}}^{\prime}(z) \quad \ldots \quad A_{n, k_{n}}^{\prime}(z)\right| \neq 0 .
$$

We thus move to proving (19).

Let us denote the order of vanishing of the function $\operatorname{det} A^{\prime}(z)$ by $m$. We define $l=m-n-\sum_{j} k_{j}$. By the definition of the $k_{j}$ we see that $0 \leq l$; furthermore, (19) is fulfilled if and only if $l=0$. We finish the proof by giving a procedure to replace $A$ by a map $\tilde{A}$ with $\tilde{l}<l$ if $0<l$.

So let us assume that

$$
\left|A_{1, k_{0}}^{\prime}(z) \quad \ldots \quad A_{n, k_{0}}^{\prime}(z)\right| \equiv 0 .
$$

By [11, Theorem II, p. 134, vol. 1, this implies that there is a nonzero polynomial $P\left(x_{1}, \ldots, x_{n}\right) \in \mathbb{C}\left[x_{1}, \ldots, x_{n}\right]$ such that

$$
P\left(A_{1, k_{0}}(z), \ldots, A_{n, k_{0}}(z)\right)=0 .
$$

Since the $A_{j, k_{j}}$ are homogeneous, we may assume that $P$ is weighted homogeneous (where $x_{j}$ has weight $k_{j}$ ), and we choose $P$ such that its weighted degree $d$ is minimal with respect to the property (21). Thus, reordering, we may assume that $P\left(A_{1}, \ldots, A_{n}\right)$ vanishes to order $d+s$, where $s>0$ and $d \geq k_{1}$, while $P_{x_{1}}\left(A_{1}, \ldots, A_{n}\right)$ vanishes to order $d-k_{1}$. Now let $\tilde{A}_{1}=P\left(A_{1}, \ldots, A_{n}\right)$, and $\tilde{A}_{j}=A_{j}$. We have

$$
\left|\tilde{A}_{1}^{\prime} \ldots \tilde{A}_{n}^{\prime}\right|=P_{x_{1}}\left(A_{1}, \ldots, A_{n}\right)\left|A_{1}^{\prime} \ldots A_{n}^{\prime}\right|,
$$

so that $\tilde{m}=m+d-k_{1}$. On the other hand, $\tilde{k}_{1}=d+s$ and $\tilde{k}_{j}=k_{j}$ for $j \geq 2$, so that $\tilde{l}=\tilde{m}-n-\sum \tilde{k}_{j}=l-s<l$ as claimed.

Combining Lemma 7 and Theorem 2 we arrive at the following. 
Proposition 8. Assume that $H, \tilde{H}$ are two automorphisms of $M$ with $j_{0}^{k} H=j_{0}^{k} \tilde{H}$ for $k \leq m_{1}-m_{0}+1$. Then $f(z, 0)=\tilde{f}(z, 0)$.

We will now determine $j_{(z, 0)}^{k} H$ from $j_{0}^{\tilde{k}} H$. Even though the technique is similar to how we obtained Proposition 8 (we will extract coefficients of power series and use the resulting equations), this step is different, as we now essentially solve linear equations. We assume that $M$ is rigid and given by $w-\bar{w}=q(z, \chi)=\sum q_{\alpha}(\chi) z^{\alpha}$.

Proposition 9. For $\alpha \in \mathbb{N}^{n}$ and $\mu \in N$ there exist rational functions $p_{\alpha, \mu}$ which only depend on $M$ such that

$$
q_{\alpha, \chi}(\bar{f}(\chi, 0)) \bar{f}_{w^{\mu}}(\chi, 0)=p_{\alpha, \mu}\left(\chi, j_{0}^{k} H, j_{0}^{k} \bar{H}, j_{(\chi, 0)}^{r} \bar{H}\right),
$$

where $k \leq|\alpha|-m_{0}+\mu+1$ and $r<\mu$.

Proof. Our starting point is equation (11), which we rewrite as

$$
\begin{aligned}
\bar{g}(0, \tau+q(z, \chi))=\bar{g}(\chi, \tau)+ & \sum q_{\alpha}(\bar{f}(\chi, \tau)) f(z, \tau+q(z, \chi))^{\alpha} \\
& -\sum q_{\alpha}(\bar{f}(0, \tau+q(z, \chi))) f(z, \tau+q(z, \chi))^{\alpha} .
\end{aligned}
$$

We are now going to deal with the extraction of the coefficient of $z^{\alpha} \tau^{\mu}$ in equation (24), and the proof consists of a double induction on $\mu$ and $|\alpha|$. We actually have (23) for $\mu=0$ in Lemma 78, so we can assume that $\mu \geq 1$ and $|\alpha| \geq m_{0}$.

Let us also remark here that $\bar{g}_{w^{l}}(\chi, 0)$ is completely determined by derivatives $\bar{g}_{w^{k}}(0)$ where $k \leq l$ and $\bar{f}_{w^{k}}(\chi, 0)$ where $k<l$. This can be seen from (24) by isolating the coefficients of $\tau^{k}$, and the proof of this fact is left to the reader.

We start by expanding the left-hand side:

$$
\bar{g}(0, \tau+q(z, \chi))=\sum_{\ell} \frac{\bar{g}_{w^{\ell}}(0)}{\ell !}\left(\tau+\sum_{\beta} q_{\beta}(\chi) z^{\beta}\right)^{\ell} .
$$

The general term coming from this sum is thus

$$
\frac{\bar{g}_{w^{\ell}}(0)}{\ell !} \prod_{j=1}^{k} q_{\beta_{j}} \tau^{\ell-k} z^{\sum \beta_{j}} .
$$

It follows that the coefficient of $z^{\alpha} \tau^{\mu}$ on the left-hand side of this equation is a polynomial in $\bar{g}_{w^{\ell}}(0)$, where $\ell=\mu+k \leq \mu+|\alpha| / m_{0} \leq \mu+|\alpha|-m_{0}+1$.

Let us now turn to the right-hand side. The first term does not contribute. We develop the second term in a power series in $(z, \tau)$. From the first factor $q_{\beta}(\bar{f}(\chi, \tau))$ of a summand we only get $\tau$ 's; only derivatives of the form $\bar{f}_{w^{\ell}}(\chi, 0)$ where $\ell \leq \mu$ can appear, and the term containing an $\bar{f}_{w^{\mu}}(\chi, 0)$ is $q_{\beta, \chi}(\bar{f}(\chi, 0)) \bar{f}_{w^{\mu}}(\chi, 0)$. Only the $\beta$ with $|\beta| \leq|\alpha|$ can appear with this term, and the terms containing $|\beta|$ with $|\beta|=|\alpha|$ are just

$$
\sum_{|\beta|=|\alpha|} q_{\beta, \chi}(\bar{f}(\chi, 0)) \bar{f}_{w^{\mu}}(\chi, 0) K_{\alpha}^{\beta}(\chi) .
$$

For the terms with $|\beta|<|\alpha|$, we use the induction hypothesis.

Expanding the other terms and the second sum on the right-hand side, it is easy to see that only terms with lower order derivatives enter. So applying the inverse matrix of $K_{\alpha}^{\beta}$, we finish the induction step. 
An induction on the result in Proposition 9. Proposition 8 and the remark at the beginning of the proof above now easily yield the following theorem.

Theorem 10. Assume that $H, \tilde{H}$ are two automorphisms of $(M, 0)$ with

$$
j_{0}^{m_{1}-m_{0}+k+1} H=j_{0}^{m_{1}-m_{0}+k+1} \tilde{H} .
$$

Then $j_{Z}^{k} H=j_{Z}^{k} \tilde{H}$ for $Z \in S_{0}$.

\section{Proof of Theorem 1}

We have now gathered all we need to prove our main theorem. Let us denote by $U \subset S_{0}$ the dense open set provided by the definition of $s$ and Lemma 6 such that $\Phi_{Z}^{s}$ is generically finite for $Z \in U$. Writing $Z^{\prime}=H(Z)=\tilde{H}(Z)$ for $Z \in S_{0}$, by Theorem 10 we have the following diagram:

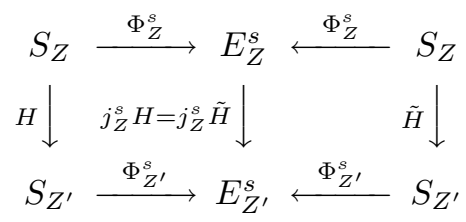

Hence, $\Phi_{H(Z)}^{s} \circ H=\Phi_{\tilde{H}(Z)}^{s} \circ \tilde{H}$ on $S_{Z}$ for $Z \in U$. But by Theorem 2 again, this implies $H=\tilde{H}$ on $S_{Z}$ for $Z \in U$; since $\bigcup_{Z \in U} S_{Z}$ contains an open set in $\mathbb{C}^{N}$, the identity principle now gives us that $H=\tilde{H}$ near 0 . The theorem is proved.

\section{REFERENCES}

1. M. S. Baouendi, P. Ebenfelt, and L. P. Rothschild, Parametrization of local biholomorphisms of real analytic hypersurfaces, Asian J. Math. 1 (1997), no. 1, 1-16. MR.1480988 (99b:32022)

2. $\longrightarrow$ CR automorphisms of real analytic manifolds in complex space, Comm. Anal. Geom. 6 (1998), no. 2, 291-315. MR1651418 (99i:32024)

3. _ Real submanifolds in complex space and their mappings, Princeton University Press, Princeton, NJ, 1999. MR1668103 (2000b:32066)

4. M. S. Baouendi, X. Huang, and L. P. Rothschild, Regularity of CR mappings between algebraic hypersurfaces, Invent. Math. 125 (1996), no. 1, 13-36. MR1389959 (97c:32028)

5. M. S. Baouendi, N. Mir, and L. P. Rothschild, Reflection ideals and mappings between generic submanifolds in complex space, J. Geom. Anal. 12 (2002), no. 4, 543-580. MR1916859 (2003m:32035)

6. M. S. Baouendi and L. P. Rothschild, Mappings of real algebraic hypersurfaces, J. Amer. Math. Soc. 8 (1995), no. 4, 997-1015. MR1317227 (96f:32039)

7. E. Cartan, Sur la géométrie pseudo-conforme des hypersurfaces de deux variables complexes I, Ann. Math. Pura Appl. 11 (1932), no. 4, 17-90.

8. - Sur la géométrie pseudo-conforme des hypersurfaces de deux variables complexes II, Ann. Scuola Norm. Sup. Pisa 1 (1932), no. 2, 333-354.

9. S. S. Chern and J. K. Moser, Real hypersurfaces in complex manifolds, Acta Math. 133 (1974), 219-271. MR0425155 (54:13112)

10. P. Ebenfelt, B. Lamel, and D. Zaitsev, Finite jet determination of local analytic CR automorphisms and their parametrization by 2-jets in the finite type case, Geom. Funct. Anal. 13 (2003), no. 3, 546-573. MR1995799 (2004g:32033)

11. W. V. D. Hodge and D. Pedoe, Methods of algebraic geometry. Vol. I, Cambridge Mathematical Library, Cambridge University Press, Cambridge, 1994, Book I: Algebraic preliminaries, Book II: Projective space, Reprint of the 1947 original. MR.1288305 (95d:14002a)

12. H. Poincaré, Les fonctions analytiques de deux variables et la représentation conforme, Rend. Circ. Mat. Palermo II. Ser. 23 (1907), 185-220.

13. N. K. Stanton, Infinitesimal CR automorphisms of real hypersurfaces, Amer. J. Math. 118 (1996), no. 1, 209-233. MR1375306 (97h:32027) 
14. N. Tanaka, On the pseudo-conformal geometry of hypersurfaces of the space of $n$ complex variables, J. Math. Soc. Japan 14 (1962), 397-429. MR0145555 (26:3086)

15. D. Zaitsev, Algebraicity of local holomorphisms between real-algebraic submanifolds of complex spaces, Acta Math. 183 (1999), no. 2, 273-305. MR1738046 (2001a:32024)

16. Unique determination of local CR-maps by their jets: A survey, Rend. Mat. Acc. Lincei, s. 913 (2002), 135-145. MR1984108(2004i:32056)

Fakultät für Mathematik, Universität Wien, Nordbergstrasse 15, A-1090 Wien, AusTRIA

E-mail address: lamelb@member.ams.org 